\title{
WOUND COMPOSITE BEAM INTERNAL STRUCTURE OPTIMIZATION
}

\author{
M. Tyburec**, J. Zeman ${ }^{* *}$, M. Lepš ${ }^{* * *}$, J. Novák $^{\dagger}$, R. Poul ${ }^{\ddagger}$
}

\begin{abstract}
A semidefinite programming formulation of truss topology optimization has been employed to design a minimum-weight internal structure of a wound composite beam, with the objective to increase its fundamental free-vibration eigenfrequency and guarantee small deflections in the compression molding load case concurrently. The optimized design was successfully manufactured by winding the additively-manufactured internal structure by a carbon/epoxy composite. Preliminary numerical results confirm the potential of the method.
\end{abstract}

Keywords: carbon fiber composites, semidefinite programming, truss topology optimization, reinforcement design, additive manufacturing

\section{Introduction}

Filament wound carbon fiber reinforced polymer matrix composites (CFRPs) are commonly used in highdemand applications, particularly because of their high stiffness-to-weight ratio, damping properties, and low sensitivity to fatigue and corrosion. Consequently, their applicability ranges from masts in marine industry and blades in wind turbines to various components in aerospace and automotive industries.

Contrary to isotropic materials such as steel or aluminum, CFRPs exhibit anisotropic behavior in general, with the largest stiffness of individual laminae aligned with the direction of carbon fibers. The effective material properties then arise from the composition and orientation of the laminate layers, and the fiberresin volume fractions.

In this contribution, we focus on a single filament wound CFRP product, a simply-supported prismatic thin-walled beam, see Fig. 1. In order to maximize its bending stiffness and maintain the low weight at the same time, its cross-section has to be as large as possible to maximize the moments of inertia, and the walls sufficiently thin. Moreover, the majority of the CFRP layers should be aligned with the longitudinal axis of the beam, and the rest used for a stabilization of the layered composite to avoid delamination. Supported by the anisotropy, such beams are, however, prone to the (shear) wall instabilities, see Fig. 2.

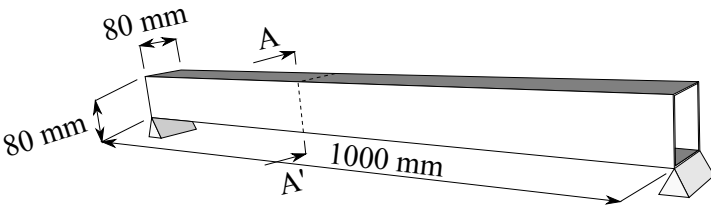

(a) Dimensions and free-vibration boundary conditions

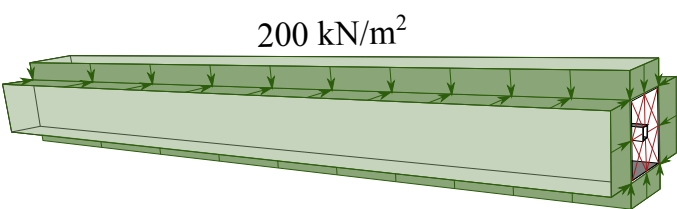

(b) Compression molding boundary conditions

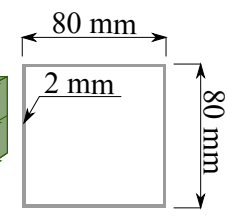

(c) Section AA'

Fig. 1: Dimensions and boundary conditions of the composite beam.

\footnotetext{
* Ing. Marek Tyburec: Department of Mechanics, Faculty of Civil Engineering, Czech Technical University in Prague, Thákurova 7/2077; 166 29, Prague; CZ, marek.tyburec@fsv.cvut.cz

** Doc. Ing. Jan Zeman, Ph.D.: Department of Mechanics, Faculty of Civil Engineering, Czech Technical University in Prague, Thákurova 7/2077; 166 29, Prague; CZ, Jan.Zeman@ cvut.cz

*** Doc. Ing. Matěj Lepš, Ph.D.: Department of Mechanics, Faculty of Civil Engineering, Czech Technical University in Prague, Thákurova 7/2077; 166 29, Prague; CZ, matej.leps@ fsv.cvut.cz

$\dagger \quad$ Ing. Jan Novák, Ph.D.: Experimental Center, Faculty of Civil Engineering, Czech Technical University in Prague, Thákurova 7/2077; 166 29, Prague; CZ, novakja@ fsv.cvut.cz

‡ Ing. Robin Poul, Ph.D.: Compo Tech PLUS, s.r.o., Nová 1316; 342 01, Sušice; CZ, robin@ compotech.com
} 


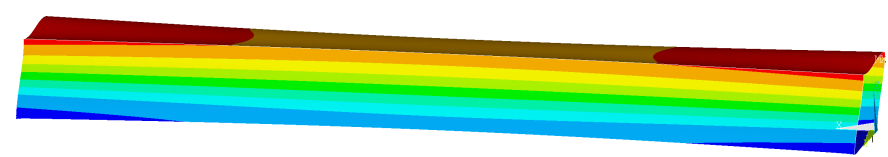

(a)

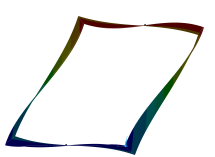

(b)

Fig. 2: (a) Side, and (b) front views on the fundamental eigenmode, $f_{0}=108.42 \mathrm{~Hz}$.

To avoid the wall instabilities, we focus here on the design of a low-weight reinforcing lattice structure, built in the beam interior, Fig. 3. The reinforcement is generated algorithmically using the truss topology optimization technique, and constrained to increase the fundamental free-vibration eigenfrequency corresponding to the shear wall instability, Fig. 2, to a prescribed value, and to keep the low weight of the beam concurrently. Moreover, the reinforcement is designed to connect temporarily the wound composite beam with a steel tube, passing through the beam interior and serving as a mount in the filament winding machine, and to withstand the compression molding pressure with limited deflections, Fig. $1 \mathrm{~b}$.

The optimized reinforcement, together with its casing, serving as the reinforcement-composite rigid connection, see Fig. 3, is printed from Acrylonitrile Butadiene Styrene (ABS) using the Stratasys 3D printer. The prototype of the beam is finalized by outer layers made up of cross-wound carbon fibers and epoxy resin, compressed subsequently, and cured.

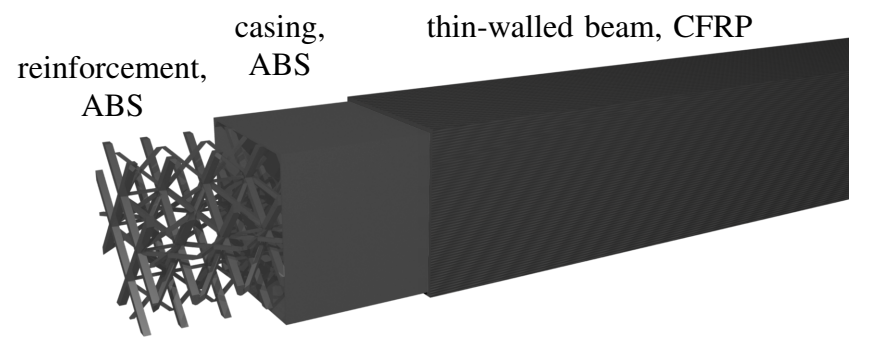

Fig. 3: Scheme of the composition of the reinforced beam.

\section{Optimal design of internal reinforcement}

The process of the composite beam design consists of three subsequent steps. First, the composition of the laminate layers is determined, and the effective properties of the transversally-isotropic material are computed using the classical lamination theory. Second, the finite element model of the composite beam is created and the beam interior discretized into a truss ground structure. For the developed model, truss topology optimization is used to determine the optimal cross-sections of individual members. Finally, manuacturability of the design is secured by post-processing of the optimization outputs.

\subsection{Finite element model}

The composite beam was modeled and discretized using the MITC4 shell elements (Bathe and Dvorkin, 1986) in MATLAB. The elements were supplied with homogenized transversally-isotropic material properties shown in Tab. 1, with the $x$ axis matching the longitudinal axis of the beam, $z$ being orthogonal to the midplane of the corresponding shell element and pointing outwards of the beam, and $y$ being orthogonal to both $x$ and $z$, generating a right-handed Cartesian coordinate system. It should be also noted that $y z$ constitutes the plane of isotropy.

The lattice ABS reinforcement of the beam interior was supplied with isotropic material properties (Cantrell et al., 2017), shown in Tab. 1. Moreover, we neglected the rotational degrees of freedoms of the lattice components, resulting in truss-like behavior. As trusses are always more compliant than the actual reinforce-

Tab. 1: Effective material properties

\begin{tabular}{ccccccc}
\hline & $E_{x}[\mathrm{GPa}]$ & $E_{y}[\mathrm{GPa}]$ & $G_{x y}[\mathrm{GPa}]$ & $\nu_{x y}[-]$ & $\nu_{y z}[-]$ & $\rho\left[\mathrm{kg} / \mathrm{m}^{3}\right]$ \\
\hline CFRP & 228.706 & 13.550 & 6.707 & 0.920 & 0.350 & 1579 \\
\hline ABS & 2.000 & 2.000 & 0.730 & 0.370 & 0.370 & 1040 \\
\hline
\end{tabular}


ment, the (finite element) model provides a reliable estimate of the structural response. This hypothesis was confirmed by comparing results of the analyses with the reinforcement modeled as truss and frame elements.

\subsection{Optimization problem formulation}

Topology optimization starts with discretization of the design domain into a set of admissible elements, and searches whether these are present in the optimal design. For topology optimization of trusses, the beam interior is discretized into $n_{\mathrm{n}}$ nodes that are interconnected by $n_{\mathrm{b}}$ bars, forming the so-called ground structure (Dorn et al., 1964). Then, we search the non-negative cross sections a of these bars, such that the total weight is minimal (1a), they increase the fundamental eigenfrequency of the composite beam to at least $\overline{f_{0}}(1 \mathrm{~b})$, and transfer the compression molding loads to the steel tube with the external work at most $c_{\mathrm{cm}}(1 \mathrm{c})$.

Quite remarkably, the problem can be represented by linear semidefinite cones (Achtziger and Kočvara, 2007), and thus it is solvable to global optimality using the convex semidefinite program:

$$
\begin{aligned}
& \min _{\mathbf{a} \in \mathbb{R}^{n_{b}}} \rho^{\mathrm{ABS}} \boldsymbol{\ell}^{\mathrm{T}} \mathbf{a}+m_{0}^{\mathrm{ABS}}+m_{0}^{\mathrm{CFRP}} \\
& \text { s.t. } \quad \mathbf{K}_{\mathrm{fv}}^{\mathrm{ABS}}(\mathbf{a})+\mathbf{K}_{0, \mathrm{fv}}^{\mathrm{CFRP}}-4 \pi^{2}{\overline{f_{0}}}^{2}\left(\mathbf{M}_{\mathrm{fv}}^{\mathrm{ABS}}(\mathbf{a})+\mathbf{M}_{0, \mathrm{fv}}^{\mathrm{CFRP}}\right) \succeq \mathbf{0} \text {, } \\
& \left(\begin{array}{cc}
c_{\mathrm{cm}} & -\mathbf{f}_{\mathrm{cm}}^{\mathrm{T}} \\
-\mathbf{f}_{\mathrm{cm}} & \mathbf{K}_{\mathrm{cm}}^{\mathrm{ABS}}(\mathbf{a})+\mathbf{K}_{0, \mathrm{~cm}}^{\mathrm{CFRP}}
\end{array}\right) \succeq \mathbf{0}, \\
& \mathbf{a} \geq \mathbf{0},
\end{aligned}
$$

in which $\mathbf{K}_{\mathrm{fv}}^{\mathrm{ABS}}, \mathbf{K}_{0, \mathrm{fv}}^{\mathrm{CFRP}}, \mathbf{M}_{\mathrm{fv}}^{\mathrm{ABS}}$, and $\mathbf{M}_{0, \mathrm{fv}}^{\mathrm{CFRP}}$ are the stiffness and mass matrices corresponding to the freevibration degrees of freedom of the $\mathrm{ABS}$ and CFRP components, respectively; $\mathbf{K}_{\mathrm{cm}}^{\mathrm{ABS}}(\mathbf{a}), \mathbf{K}_{0, \mathrm{~cm}}^{\mathrm{CFR}}$, and $\mathbf{f}_{\mathrm{cm}}$ denote the stiffness matrices of the ABS and CFRP parts, and the force vector for the degrees of freedom in the compression molding load case. Further, $\rho^{\mathrm{ABS}}$ constitutes density of the printed internal structure, $\ell$ is a vector of the lengths of all bars, and $m_{0}^{\mathrm{ABS}}$ and $m_{0}^{\mathrm{CFRP}}$ are the masses of the printed casing, and of the composite beam, respectively. The notation " $\mathbf{A} \succeq \mathbf{0}$ " represents a linear matrix inequality (LMI), requiring positive semidefiniteness of the matrix $\mathbf{A}$.

In this contribution, we used a modular ground structure generated from repeated blocks, Fig. 4, to maintain a low number of design variables, and also support-less printing due to the inclined angles greater than $35^{\circ}$. We also required $\overline{f_{0}}=300 \mathrm{~Hz}$, and computed $c_{\mathrm{cm}}$ such that the maximum displacement is at most $0.5 \mathrm{~mm}$.

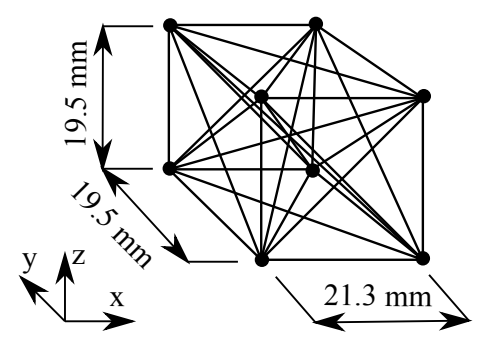

Fig. 4: Representative block of the ground structure repeated throughout the beam interior.

The optimization program contained 10, 216 potential bars and two LMIs of the respective sizes $7,170 \times$ 7,170 and $5,761 \times 5,761$. Its solution required 42 core hours using the Mosek (2017) optimizer on an Intel ${ }^{\circledR}$ Xeon ${ }^{\circledR}$ E5-2650v4 processor at the MetaCentrum distributed computing cluster.

\subsection{Post-processing}

Despite the computed design being globally optimal with respect to the predefined ground structure, the formulation (1) does not guarantee neither beam manufactureability, nor kinematic stability. As we handle these aspects in a post-processing step, we lose the true optimality and obtain a nearly-optimal design.

In order to provide stable and manufactureable internal structure, individual positive cross-sections were rounded up to the nearest square side length divisable by $0.4 \mathrm{~mm}$ in order to match the printer resolution. Moreover, rounding was also used to guarantee slenderness smaller than 40 . 


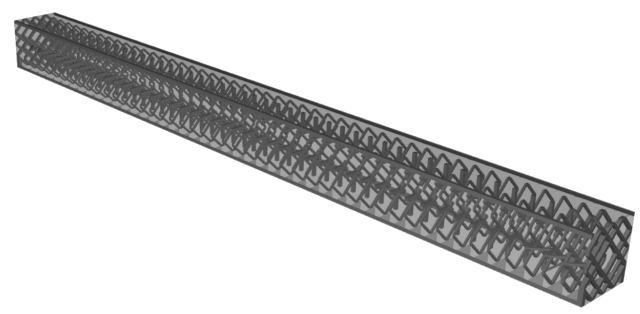

(a) CAD model of reinforcement

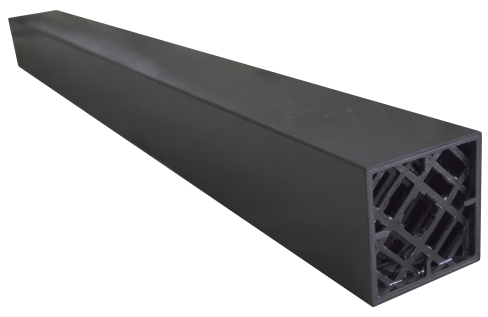

(b) Manufactured prototype

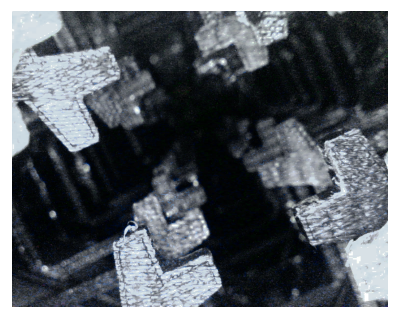

(c) Reinforcement detail

Fig. 5: Optimized design of the reinforced composite beam.

Rigid connection among the composite beam and the ABS reinforcement is secured by $0.8 \mathrm{~mm}$ thin printed casing, recall Fig. 3. The steel tube-reinforcement connection was provided by printed L-shaped handles, see Fig. 5c. Finally, economic support-less printing is achieved by splitting the reinforcement into $21.3 \mathrm{~mm}$ wide segments, connected by means of acetone etching subsequently.

\section{Results}

The internal reinforcement was printed, glued, and the beam wound and cured successfully, Fig 5. The optimization and post-processing increased the beam weight by $48.92 \%$ from $984 \mathrm{~g}$ to $1465 \mathrm{~g}$, and also the fundamental eigenfrequency by $124.31 \%$ from $108.42 \mathrm{~Hz}$ to $243.20 \mathrm{~Hz}$. The fundamental eigenmode of the optimized design exhibits combined shear and bending vibrations, see Fig. 6. Contrary to the requirements and due to the coarse discretization, matching the ground structure in the optimization, the actual value of the fundamental eigenfrequency on fine mesh is by $18.93 \%$ lower than the prescribed value.

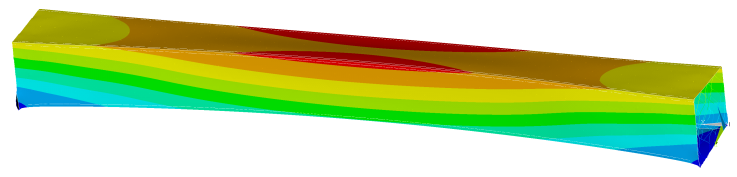

(a) Side view

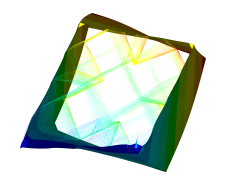

(b) Front view

Fig. 6: Views on the fundamental eigenmode of the reinforced beam, $f_{0}=243.20 \mathrm{~Hz}$.

\section{Conclusions}

In this contribution, we have outlined an approach for a conceptual design of an internal reinforcement of a simply-supported composite beam. The numerical results suggest that the approach was successful in increasing the beam fundamental eigenfrequency, albeit by a smaller amount compared to the requirement. Additional insides shall be given by experimental validation of the manufactured prototype.

\section{Acknowledgments}

This work was supported partly by the Grant Agency of the CTU in Prague, SGS18/036/OHK1/1T/11, and by the Technology Agency of the Czech Republic, TAČR TH02020420. Access to computing and storage facilities owned by parties and projects contributing to the National Grid Infrastructure MetaCentrum provided under the programme "Projects of Large Research, Development, and Innovations Infrastructures" (CESNET LM2015042), is also greatly appreciated.

\section{References}

Achtziger, W., Kočvara, M. (2007). Structural topology optimization with eigenvalues. SIAM Journal on Optimization, 18(4), 1129-1164.

Bathe, K. J., Dvorkin, E. N. (1986). A formulation of general shell elements-the use of mixed interpolation of tensorial components. International Journal for Numerical Methods in Engineering, 22(3), 697-722.

Cantrell, J. T., Rohde, S., Damiani, D., Gurnani, R., DiSandro, L., Anton, J., Ifju, P. G. (2017). Experimental characterization of the mechanical properties of 3D-printed ABS and polycarbonate parts. Rapid Prototyping Journal, 23(4), 811-824.

Dorn, W. S., Gomory, R. E., and Greenberg, H. J. (1964). Automatic design of optimal structures. Journal de Mecanique, 3:25-52.

Mosek, A. P. S. (2010). The MOSEK optimization software. Online at http://www. mosek. com. 